\title{
Immunological control of coccidiosis in poultry
}

\author{
Kayla Price and John R. Barta* \\ *This study was conducted under the supervision of Professor John R. Barta, \\ Department of Pathobiology, Ontario Veterinary College, \\ University of Guelph, Guelph, Ontario, Canada
}

\begin{abstract}
Coccidiosis - caused by parasites of the genus Eimeria - is an infection known to damage poultry. Eimeria spp. follow a typical coccidian lifestyle consisting of an endogenous asexual and sexual phase and an exogenous infectious phase. Eimeria spp. infections are self-limiting because the number of asexual, pathogenic, cycles is genetically predetermined. However, the infection may be chronic because of oocyst cycling in feces. Coccidiosis is a burden on the poultry industry and many preventive anticoccidial drugs have been created; however, a major limit is emerging drug resistance. Live vaccination is another method of coccidiosis prevention where protective immunity is developed by a dose of non-attenuated Eimeria spp. that is then cycled in the feces to produce protective immunity. The cycling of the controlled infection with live vaccination of replacement laying hens is influenced by commercial caging techniques. This review provides an overview of coccidiosis caused by Eimeria species infecting domestic fowl and the nature of coccidial infections in commercial poultry houses.
\end{abstract}

$\mathrm{C}$ occidiosis is a long-recognized infection known to cause damage in a variety of animal species. This literature review will provide an overview of coccidiosis caused by Eimeria species infecting the domestic fowl and the nature of coccidial infections in commercial poultry houses. The first section examines the parasite, various Eimeria spp. and their pathogenicity. Subsequent sections focus on the effect on the industry, anticoccidial and vaccination prevention methods as well as housing techniques to induce protective immunity.

\section{EIMERIA AND COCCIDIOSIS}

\section{Eimerian lifecycle}

Eimeria species are members of the protistan phylum Apicomplexa. All are monoxenous parasites that infect one or a few closely related vertebrate hosts [1]. Eimeria spp. follow a typical coccidian lifecycle with both exogenous (outside of the host) and endogenous (inside of the host) developmental stages. The general lifecycle is commonly identified in literature; however, the specific processes vary slightly from one species to the next.

A typical lifecycle of an Eimeria sp. is illustrated in Figure 1[2]. Unsporulated oocysts are released to the environment via the feces of poultry. The unsporulated oocyst is non infective; however, after approximately 48 hours at optimum temperature (around $30^{\circ} \mathrm{C}$ ) and oxygen tension the oocysts sporulate [3]. The sporulated oocyst is tetrasporic and dizoic with four sporocysts that contain two infective sporozoites within them [1]. These infective oocysts can remain viable in the litter for an extended period (months to years under ideal conditions) until transmitted fecal-orally to a definitive host.

The endogenous phase of the eimerian life-cycle is initiated when a bird has ingested a sporulated oocyst [1]. The grinding action of the gizzard releases the sporocysts from the oocyst, while enzymatic action of the upper intestinal tract releases the sporozoites from the sporocysts [4]. Sporozoites infect intestinal host epithelial cells in the gut lining in various regions depending on the species of Eimeria [3]. Within the host intestinal epithelial cells the sporozoite forms a trophozoite that undergoes merogony, asexual divisions, to form an immature meront (schizont) [1]. The cytoplasm of the meront divides to form merozoites [4]. Merozoites lyse out of the original infected intestinal epithelial cells to infect new epithelial cells completing a second cycle of merogony [5]. It should be noted that the lifecycle of each species of Eimeria has a predetermined number of cycles of merogony that lead to characteristic prepatent periods [6,3]. The predetermined number of cycles ranges between two and four. At the conclusion of the last cycle of merogony the resulting merozoites enter new host intestinal epithelial cells and initiate gametogony, the sexual phase of the lifecycle [1]. Within gametogony the merozoites either become microgamonts that form microgametes, male gametes, or they become macrogamonts that mature to female macrogametes [4]. Merozoites that form a microgamont undergo multiple divisions that ultimately result in the production of many biflagellate microgametes, whereas merozoites that form a macrogamont mature to a single large macrogamete [4]. Microgametes will invade macrogamete infected intestinal host epithelial cells [4]. The 


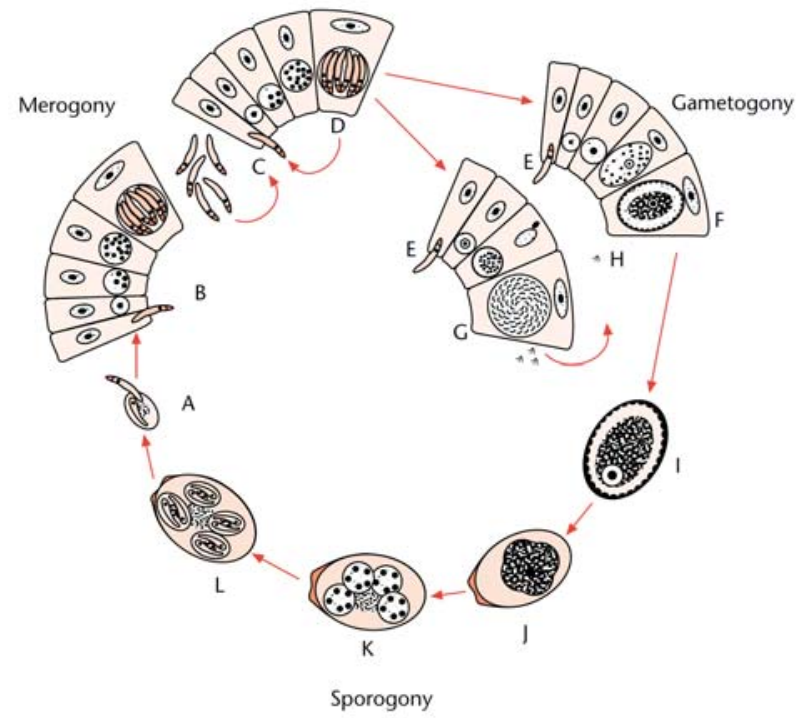

Figure 1. The life cycle of a typical Eimeria species. Sporozoites (A) excyst from sporocysts in the intestinal tract of Gallus gallus domesticus. Sporozoites infect an intestinal host epithelial cell and initiate merogony (B). Merozoites (schizonts) exit and infect neighbouring intestinal host epithelial cells for a genetically determined number of merogonic cycles (C-D). After the genetically determined number of cycles, merozoites undergo gametogony (E) producing microgametes (F) and macrogametes (G). Motile microgametes locate and fertilize a mature macrogamete producing a zygote $(\mathrm{H})$. The unsporulated oocyst, zygote, is shed in the feces (I). Sporogony occurs in the environment to produce a sporulated, infective oocyst (I-L). (From Barta, 2001) [2].

now fertilized macrogamete (a zygote) quickly forms an oocyst wall becoming an unsporulated oocyst that will be shed in the feces [1].

Nine species of Eimeria are known to infect Gallus gallus domesticus at various locations along the intestinal tract: E. acervulina, E. brunetti, E. hagani, E. maxima, E. mitis, E. mivati, E. necatrix, E. praecox and E. tenella. Of these nine species only seven are considered pathogenic to poultry: E. acervulina, E. brunetti, E. maxima, E. mitis, E. necatrix, E. praecox and E. tenella [7]. The pathogenic species have a cosmopolitan distribution and can cause infection simultaneously. Thus, disease caused by Eimeria species better represents a disease complex [7].

\section{Avian Coccidiosis}

Unlike many pathogens, the severity of coccidiosis in immunologically naïve birds is directly related to the number of infective stages that are ingested [8]. For example, oocysts ingested in the thousands will produce overt clinical signs in naïve birds, whereas oocysts ingested in the hundreds may only produce subclinical infections [8]. This relationship between number of oocysts ingested and resulting severity of

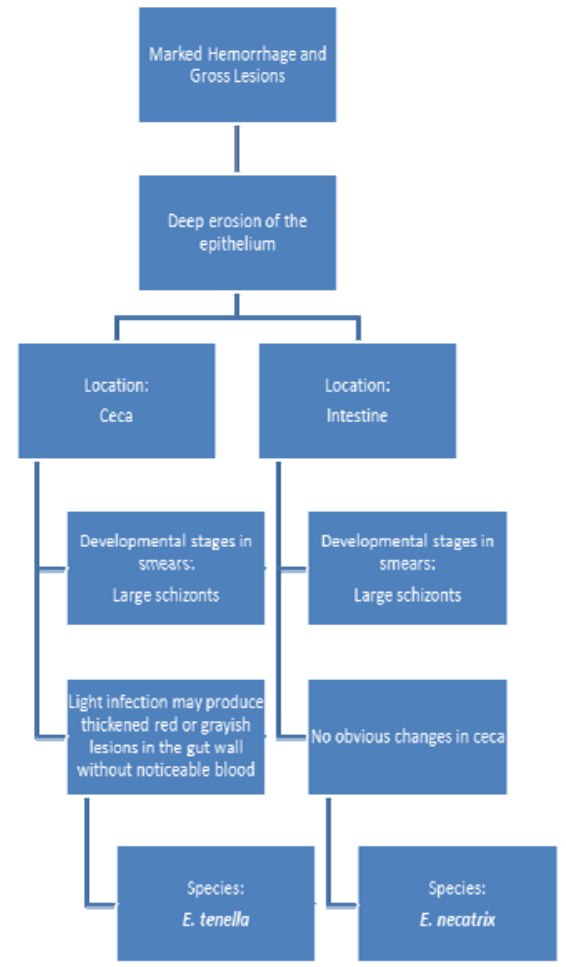

Figure 2. Simple diagnostic key for identification of species of Eimeria in chickens demonstrating marked hemorrhages (Modified from Reid and Long, 1979) [2].

infection occurs because Eimeria spp. produce self-limiting infections with a genetically predetermined number of asexual, pathogenic, cycles. Avian coccidiosis is categorized into three forms of infection severity: 1) clinical coccidiosis demonstrating morbidity in the form of bloody diarrhea and, less commonly, mortality; 2) subclinical coccidiosis that is not immediately obvious through noticeable clinical signs but manifests as decreased weight gain, feed conversion efficiency and egg production in layer hens; and 3) coccidiasis that represents a mild interaction between host and parasite with no detectable adverse affects on the host [9, 10]. Reid [8] found that clinical coccidiosis was displayed in birds in close confinement as these birds are exposed to a large number of infective oocysts. Conversely, subclinical coccidiosis was associated with free range managed flocks for the suggested reason that oocysts were spread around in a large area decreasing the potential of the definitive host to ingest similarly large numbers of infective oocysts [8]. Ruff [11] determined that each oocyst ingested by a host has the potential to give rise to hundreds of thousands of oocysts within the feces after seven to twelve days.

Even though Eimeria spp. are reproductively selflimiting parasites, Vetterling [12] discovered that an infection from this parasite could be labeled as chronic; however, the chronic nature was derived from re-infection through cycling of oocysts in the feces. If cycling is prevented then upon cessation of the asexual cycles 


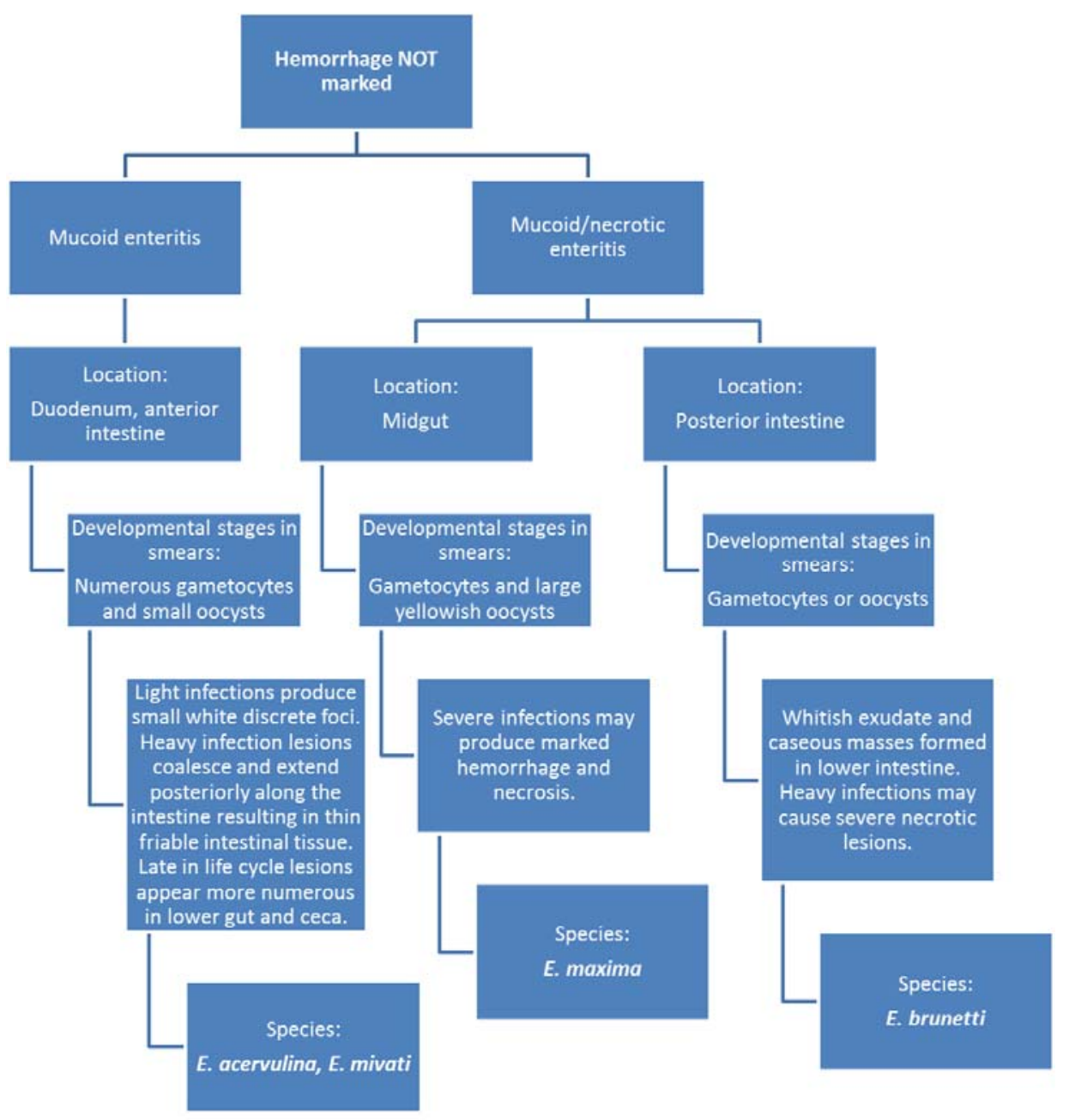

Figure 3. Simple diagnostic key for identification of species of Eimeria in chickens with no marked hemorrhages (Modified from Reid and Long, 1979) [2].

multiplication and oocyst production will cease and the bird is no longer infected or affected by the parasite [8]. In this way, infection with a small number of oocysts is self limiting as there is a lower chance for recycling of sporulated oocysts but a heavy dose of oocysts are "chronic" in that there is a higher chance of cycling and re-infection [8]. On the other hand, Bhanushi and Long [13] suggested that infection with Eimeria may suppress host immune responses to pathogenic antigens making the host vulnerable to other diseases as well as infection with other strains and species of Eimeria. Since there is no stimulation of cross immunity between species of coccidia it is possible to have several and/or concurrent outbreaks of different Eimeria species in one flock [14].

\section{Diagnosis}

Eimeria species vary in infection location and it is this location that depends on the species of the parasite [3]. The infection location ranges from the duodenum to the cecum and the degree of pathogenicity ranges from mild to severe depending on the species of the parasite (Figure S1) [3]. Williams [7] reported some species of Eimeria (E. mitis and $E$. praecox) reduce growth rate and feed efficiency whereas others may cause severe morbidity with the aforementioned symptoms and bloody diarrhea or mortality may ensue ( $E$. necatrix and E. tenella) (Figures 2 and 3 ).

Reid and Long [3] established that oocysts of Eimeria species cannot be distinguished simply using morphology. The oocysts are categorized according to size with minute variations in length and width into the categories small, medium and large [3]. The small size category is made up of E. acervulina, E. mivati and E. mitis; the medium size category includes E. necatrix, E. tenella, E. praecox and E. brunetti; and the large size category contains E. maxima (Figure S1) [3]. A tentative diagnosis can be made based on the general characteristics of any lesions and their location(s) regardless whether oocysts are being shed or not (Figures 2 
and 3) [3]. For the most part, knowing the specific Eimeria species causing coccidiosis in chickens is of little practical significance because control measures (management and medications) are largely identical for all species.

\section{THE POULTRY INDUSTRY AND ANTICOCCIDIAL DRUGS}

\section{The Poultry Industry and Coccidiosis}

Coccidiosis is encountered wherever chickens are reared and this disease has generated an immense burden on the poultry industry. The poultry industry raises approximately 40 billion chickens annually and coccidiosis is the most frequently reported disease of chickens worldwide [6,7]. Lee et al [15] suggested this disease has had the greatest impact on poultry production while Dalloul and Lillehoj [16] determined that in-feed medication for prevention and treatment of this infection account for the major portion of the economic burden. However, Ruff [11] claimed that the economic loss annually exceeding $\$ 1.5$ billion US is from a multitude of factors including decreased weight gain, decreased feed efficiency, decreased egg production in addition to the cost of treatment for the infection. It was determined in 1997 that a decrease in feed efficiency where $0.01 \mathrm{~kg}$ more feed is needed per one kilogram weight gain has the potential to cost the poultry industry $\$ 70$ million US [11].

Coccidiosis is a disease that is difficult to eradicate as the oocyst wall is environmentally resistant. It has been found that an unsporulated oocyst can survive up to seven months in the cecal tissue and the sporulated oocyst can survive up to 602 days in the exogenous environment [8]. Oocysts are resistant to the variety of disinfectants used in commercial houses; thus, these environmentally resilient oocysts can be transmitted among farms through movement of personnel or equipment $[8,11]$.

\section{Anticoccidial drugs}

Anticoccidial drugs were the treatment of choice in the poultry industry as large volume manufacturing methods and market competition brought the cost of treatment to less than $\$ 0.01$ US per bird [14]. The use of anticoccidial drugs allowed for more birds to be reared in a single house [14]. Most anticoccidial drugs must be removed from the medicated feed approximately a week before marketing due to both regulations and cost effectiveness [14]. Legislation that prohibited the use of medication until slaughter was introduced because of concern that residues from the drugs may contaminate the poultry and be toxic for human consumption [10]. However, if the drug is removed then the bird is susceptible to infection because there may be no protective immunity acquired while the bird is on anticoccidial drugs; any infective oocysts in the litter may thus cause a severe infection [8]. In this way, legislation produced a withdrawal period whereby there is a maximum risk of coccidial infection.
A major limitation of the use of prophylactic anticoccidials is the emergence of drug-resistant strains after a drug has had prolonged use in the field [14]. To combat the resistance, shuttle and rotation systems have been employed [14]. In the shuttle program the different drugs are used during one period of juvenile growth to market size (growout); whereas, in the rotation program the type of drug is switched after one or several grow-outs or seasonally [17]. However, Lee et al [15] discovered that careful use of a shuttle or rotation program does not fully prevent drug resistance but does increase the useful longevity of the drug. Some of the new ionophorous antibiotic anticoccidial drugs such as monensin or lasalocid, had been used for some time in the field before, inevitably, drug resistant parasites emerged [18]. Development of new anticoccidial drugs are costly due to stringent testing requirements and few, if any, are being developed for market [16].

\section{ACQUIRED IMMUNITY AND VACCINATION}

\section{Acquired Immunity and Vaccination}

All birds can acquire immunity against the various species of Eimeria, however what cellular mechanisms underlie this protective immunity is beyond the scope of this paper. It was discovered that daily inoculations of small numbers of oocysts over twenty days produced a stronger immunity than when a large number of oocysts was given in a single dose [8]. This discovery was labeled as a trickle infection. The trickle infection suggests that for chickens Eimeria species protective immunity is developed only after the bird has been infected by the parasite several times through cycling. In a literature review authored by Beattie [19] it was generally found that the motile sporozoites played an important role in conferring an immune response while the gametogonic stages elicited little protective immunity. Protective immunity is generally regarded as the prevention of oocyst production and absence of clinical signs in birds challenged by the parasites [19]. Once protective immunity is developed for a certain Eimeria species (and sometimes strain) the bird is immune against further infection with this parasite [20]. Eimeria maxima best demonstrates acquired immunity; a single infection initiated with only a few oocysts results in the development of almost complete (>99.99\%) immunity [21]. However, various studies have shown that immunity against one strain of pathogen will not necessarily protect against a different strain of the same species; this has been demonstrated with the antigenetically variable Eimeria maxima as well as Eimeria acervulina [5, 18, 20, 21]. Conversely, it was shown that some strains of birds have innate genetically controlled resistance to some Eimeria species but this is not profitable as a complete protection against coccidiosis is not demonstrated [8].

The maximum natural challenge of oocysts to birds usually occurs between three and five weeks of age, thus it is necessary to initiate immunity before this challenge occurs [22]. The objective of vaccination is to induce an immune 
Table 1. Live anticoccidial vaccines developed for use in chickens. Various live attenuated and non-attenuated anticoccidial vaccines developed for commercial broilers, layers, breeders and caged chickens (Adapted from Williams, 2002; Vermeulen et al., 2001) [9, 10].

\begin{tabular}{|c|c|c|c|c|c|}
\hline Trade Name & Bird Type & Species & Attenuated & Administration Route & Age of chicks \\
\hline Coccivac $^{\circledR}-B$ & $\begin{array}{l}\text { Heavy } \\
\text { broilers }\end{array}$ & E. acervulina, E. maxima, E. mivati, E. tenella & No & $\begin{array}{l}\text { Hatchery spray, ocular, water, } \\
\text { feed spray }\end{array}$ & Single dose at 1 to 14 days \\
\hline Coccivac $^{\circledR}-D$ & $\begin{array}{l}\text { Breeders/ } \\
\text { layers }\end{array}$ & $\begin{array}{l}\text { E. acervulina, E. brunetti, E. hagani, E. } \\
\text { maxima, E. mivati, E. necatrix, E. praecox, } \\
\text { E. tenella }\end{array}$ & No & $\begin{array}{l}\text { Hatchery spray, ocular, water, } \\
\text { feed spray }\end{array}$ & Single dose at 1 to 14 days \\
\hline ImmucoX ${ }^{\circledR} \mathrm{C1}$ & $\begin{array}{l}\text { Broilers, } \\
\text { roasters }\end{array}$ & $\begin{array}{l}\text { E. acervulina, E. maxima, E. necatrix, } \\
\text { E. tenella }\end{array}$ & No & Water, oral gel & Single dose 1 to 4 days \\
\hline Immucox ${ }^{\circledR} \mathrm{C2}$ & $\begin{array}{l}\text { Breeders, } \\
\text { layers }\end{array}$ & $\begin{array}{l}\text { E. acervulina, E. brunetti, E. maxima, } \\
\text { E. necatrix, E. tenella, E. mivati, E. praecox }\end{array}$ & No & Water, oral gel & Single dose 1 to 4 days \\
\hline Livacox $^{\circledR} D$ & $\begin{array}{l}\text { Caged } \\
\text { chickens }\end{array}$ & E. acervulina, E. tenella & Yes & Water & Single dose at 1 to 10 days \\
\hline Livacox ${ }^{\circledR} T$ & $\begin{array}{l}\text { Broilers, } \\
\text { breeders }\end{array}$ & E. acervulina, E. maxima, E. tenella & Yes & Water, ocular & Single dose at 1 to 10 days \\
\hline Livacox $^{\circledR} Q$ & broilers & $\begin{array}{l}\text { E. acervulina, E. brunetti, E. maxima, } \\
\text { E. tenella }\end{array}$ & Yes & Water, ocular & Single dose at 1 to 10 days \\
\hline ParacoX $^{\circledR}$ & $\begin{array}{l}\text { Broilers, } \\
\text { breeders, } \\
\text { layers }\end{array}$ & $\begin{array}{l}\text { E. acervulina, E. brunetti, E. maxima (two } \\
\text { antigenically different strains), E. mivati, } \\
\text { E. necatrix, E. praecox, E. tenella }\end{array}$ & Yes & Water, feed spray & Single dose at 1 to 9 days \\
\hline Paracox $^{\circledR}-5$ & Broilers & $\begin{array}{l}\text { E. acervulina, E. maxima (two antigenically } \\
\text { different strains), E. mivati, E. tenella }\end{array}$ & Yes & $\begin{array}{l}\text { Hatchery spray, water, feed } \\
\text { spray }\end{array}$ & Single dose at 1 or 3 days \\
\hline$V A C M^{\circledR}$ & Broilers & E. maxima (ionophore resistant) & No & Beak-o-Vac ${ }^{\circledR}$ machine & Single dose at day-old \\
\hline Nobilis COX ATM & $\mathrm{n} / \mathrm{a}$ & $\begin{array}{l}\text { E. acervulina, E. tenella, E. maxima } \\
\text { (two antigenically different strains) }\end{array}$ & No & $\begin{array}{l}\text { Drinking water, hatchery } \\
\text { spray }\end{array}$ & $\mathrm{n} / \mathrm{a}$ \\
\hline
\end{tabular}

response that is adequate to enable birds to resist a challenge with virulent parasites [22]. Newly hatched birds are not fully susceptible to coccidial infection due to high maternal antibodies [6, 19]. Additionally, excystation of oocysts in newly hatched birds is inefficient due to relatively lower total chymotrypsin activity and total bile salts in the intestinal tract $[6,19]$. Thus, a vaccine must be introduced early in the bird's life so cycling of oocysts can occur and protective immunity generated.

There are three main features of coccidial infections in chickens that support the use of live vaccination for the control of coccidiosis in large commercial poultry operations [6]. Firstly, Eimeria is a self-limiting infection whereby a defined number of asexual cycles limits infectivity and only cycling of large numbers of oocysts can produce severe reinfection [6]. Secondly, birds are less susceptible to infection at a younger age thus soon after hatching the birds can be inoculated and immunity can develop with less risk of severe pathogenesis [6]. Thirdly, immunity can be produced against a homologous species of Eimeria, generating acquired immunity similar to that elicited by a trickle infection with limited to no pathogenesis [6]. Properly executed, vaccination could confer protective immunity at three to four weeks of age when a non-vaccinated bird reared using anticoccidial drugs would be most susceptible to infection $[10,14]$. Therefore, the literature demonstrates that vaccination as a means of coccidiosis prevention is a longterm solution that can be used on a wide commercial scale and is not limited by drug-resistance. Although there is the risk of the appearance of antigenically distinct strains of Eimeria spp. that will not be controlled by the vaccine strains of these parasites, live non-attenuated vaccines can be easily modified by incorporating any immunological variants into updated vaccine formulations.

\section{Comparison of Vaccine Types}

There are many types of coccidiosis vaccines being formulated; however, the literature defined two variations that are commonly used in commercial houses: live oocyst non-attenuated and live oocyst attenuated (Table 1). Lightowlers [20] described live oocyst attenuated vaccines as an avirulent vaccine that demonstrated reduced pathogenicity while maintaining the ability to induce protective immunity. McDonald and Shirley [6] as well as Dalloul and Lillehoj [16] suggested that the industry attraction to this type of vaccination is for the reason that protective immunity can be induced without prospect of the occurrence of clinical disease. Non-attenuated strains are selected via passage through embryonated hen egg or by selection for precocity [9]. Major disadvantages have been presented in literature regarding this form of vaccine $[5,6,9,15]$. McDonald and Shirley [6], Dalloul and Lillehoj [16] and Innes and Vermeulen [10] determined that the production costs were high due to the need for specific pathogen free chickens for vaccine development and production. Moreover, Williams [9] determined that these vaccines may not consistently provide a satisfactory combination of immunogenicity and attenuation of virulence. Live oocyst non-attenuated vaccines are defined as vaccinations which introduce a controlled number of oocysts of one or more wild-type Eimeria species to induce protective immunity [5]. Like all live vaccines, Innes and Vermeulen [5] noted that this form of vaccine has a limited shelf-life; however, live coccidiosis vaccines would be in steady demand (thus shelf life becomes 
Table 2. Administration methods of anticoccidial vaccines. Various administration methods and their descriptions for anticoccidial vaccines delivered to commercial chickens (Adapted from Williams, 2002) [9].

\begin{tabular}{|l|l|}
\hline Administration Method & Description \\
\hline Intra-ocular & $\begin{array}{l}\text { Sprayed into the eye of a chicken whereby the } \\
\text { oocysts pass down the nasolachrymal duct to } \\
\text { reach the intestine via the buccal cavity }\end{array}$ \\
\hline Hatchery Spray & $\begin{array}{l}\text { Coloured vaccine formulation is sprayed over } \\
\text { trays of chicks in the hatchery and oocysts } \\
\text { taken up by direct oral routes, ocular routes } \\
\text { and indirectly through preening }\end{array}$ \\
\hline Edible gel & $\begin{array}{l}\text { Coloured gel trays that can be placed on the } \\
\text { feed or in the water }\end{array}$ \\
\hline Spray-on-feed & $\begin{array}{l}\text { An aqueous suspension of vaccine oocysts } \\
\text { that can be sprayed onto the first feed that } \\
\text { chicken receive after placement in houses }\end{array}$ \\
\hline Intra-yolk sac & $\begin{array}{l}\text { A recently discovered route whereby a device } \\
\text { delivers live vaccine by intra-yolk sac injection } \\
\text { of hatchling chicks }\end{array}$ \\
\hline
\end{tabular}

less of an issue) and a relatively modest number of chickens could provide enough live oocyst vaccine material to inoculate 40 billion broilers (meat birds) per year. In addition, Lee et al [15] stated that the cost of production for this vaccine was similar to that of the production of anticoccidials and performance was equivalent. In contrast, Dalloul and Lillehoj [16] stated that the live oocyst nonattenuated vaccine may not contain sufficient numbers of more pathogenic species thus the production of protective immunity is solely dependent on reinfection from cycling of oocysts resulting from the immunizing dose. Infected birds regularly shed oocysts in the feces thus a technique to enhance recycling of oocysts may provide the enhancement needed to optimize the use of these live vaccines. Despite the complexity of generating live coccidiosis vaccines, studies are still being conducted to optimize this strategy through production of a vaccine with a long shelf-life that addresses antigenic variation and remains relatively inexpensive to manufacture $[5,6,16,20]$.

\section{CocCIVAC $^{\circledR}$}

\section{Coccivac $D^{\circledR}$ live oocyst vaccine}

Coccivac $\mathrm{D}^{\circledR}$, developed in the 1950s, was the first commercial live oocyst non-attenuated vaccine [6]. This formulation of vaccine contains oocysts of the seven aforementioned wild-type Eimeria species affecting commercial poultry (Table 1) [6]. Coccivac $D^{\circledR}$ requires both uniform distribution of oocysts and sufficient cycling of infective oocysts $[6,16]$. Failure to produce satisfactory protective immunity may be a result of a failure to ingest a sufficient number of vaccine oocysts and/or poor litter management [8, 20]. The combination of poor litter management and insufficient inoculum ingestion promotes a situation where a bird does not acquire protective immunity and is subjected to a high challenge dose of virulent oocysts that correlates with high pathogenicity. Thus, the efficacy of this vaccine is limited by the administration methods and caging techniques to promote oocyst cycling.

\section{Administration Methods}

An extensive summary of commercial vaccination administration methods was provided by Williams [9] and is summarized in Table 2. Currently, the most common method of administration of Coccivac $\mathrm{D}^{\circledR}$ in commercial houses of the United States is via hatchery spray [9]. In this form of administration the oocysts are suspended in coloured water and sprayed over the chicks in a cage setting [16]. The colour promotes preening as well as providing the ability of the hatcheries to visually evaluate the success of inoculate ingestion [16].

\section{Caging techniques and oocyst cycling}

The commercial poultry industry generates chickens for both meat (broiler) and egg (layer) production; however the housing methods for these birds are different thus cycling of oocysts is not the same. In commercial broiler housing, birds are grown on the bedding-covered floor of large rooms that have suspended nipple waterers and feeding stations. Feces accumulate on the floor as the birds grow and as a result there is considerable opportunity for oocyst cycling within the house. However, replacement layer hens (pullets) are frequently reared in elevated, multi-tiered cages before transfer to a layer house. The cages that house the birds are manufactured so the wire mesh floor has $1 \mathrm{~cm}$ or larger spacing to allow droppings to fall out of reach of the birds [14]. A reason for the employment of this form of caging is for cleanliness and prevention of ingesting feces and other potential pathogenic organisms [14]. McDougald [14] and Reid [8] affirm that complete sterility from infective oocysts is counterproductive as no subclinical infection can occur. No protective immunity may develop in such a situation and upon transfer to a different form of commercial housing the bird is at risk for clinical coccidiosis. However, Reid [8] cautions that dense confinement of birds permits the rapid accumulation of oocysts and clinical coccidiosis may occur. The ideal environment will provide a small number of sporulated oocysts that produces a trickle infection known to elicit protective immunity [8]. It is evident in the literature that vaccination provides one exposure to sporulated oocysts but to obtain protective immunity the vaccine oocysts must be recycled in the feces to produce a trickle infection [5, 8, 9, $10,16]$. Oocysts shed in the feces of vaccinated chicks must be exposed to optimal moisture, warmth and oxygen for 36 to 72 hours before the oocyst becomes sporulated in the environment and thus infective to other birds [9]. Williams [9] studied flock density and determined that a high density flock provided more litter turnover thus better oxygen requirements for oocyst sporulation. In addition, Williams [9] suggested that high flock density induced stress in the birds and stress correlated with enhanced immune resistance to certain diseases, such as coccidiosis. Thus, a high density flock may allow for a rapid accumulation of oocysts, 
increased rates of sporulation and higher stress responses which may be beneficial in moderation or harmful if in excess; however, more definitive studies need to be conducted $[8,9]$.

As previously mentioned the commercial wire caging housing itself will not permit adequate oocyst cycling as the feces fall away before cycling occurs; however, some feces may ricochet into the lower cages of multi-tiered systems. These birds then have an increased exposure to the parasite and may develop a stronger protective immunity. It is postulated that sufficient managerial factors in combination with commercial housing that favours cycling will induce protective immunity.

\section{CONCLUSION}

This literature review demonstrated the major effect Eimeria infections have on the poultry industry and the methods employed to provide protective immunity. Currently the literature has not demonstrated any study that has shown a means to eradicate Eimeria from commercial houses [8]. Anticoccidial drugs commonly used in the industry have suffered from an increase in the occurrence of drug-resistant strains, in spite of shuttle or rotation techniques implemented to extend the effective commercial lives of these products [15]. Live attenuated or non-attenuated coccidiosis vaccines may offer a realistic long term solution to the coccidiosis problem [10]. However, successful use of live oocyst attenuated and non-attenuated vaccines is dependent on housing techniques that balance oocyst cycling (providing protective immunity) with moderate numbers of infective oocysts in the housing environment (limiting pathogenic effects on the birds).

\section{ACKNOWLEDGMENTS}

This research was supported through grants from the Natural Sciences and Engineering Research Council of Canada (NSERC) and the Ontario Ministry of Agriculture, Food and Rural Affairs (OMAFRA) to JRB. I would like to express my appreciation of the support provided by Julie Cobean and Mosun Ogedengbe.

\section{REFERENCES}

1. Fayer, R. (1980). Epidemiology of protozoan infections: the coccidia. Veterinary Parasitology, 6, 75103.

2. Barta, J.R. (2001). Coccidiosis. Encyclopedia of Life Sciences, $19 \quad$ April 2001, 1-8. DOI: 10.1038/npg.els.0001947

3. Reid, W.M. \& Long, P.L. (1979). A diagnostic chart for nine species of fowl coccidian. In N.B. Bowen (Ed), Georgia Agricultural Experiment Stations Technical Bulletin (pp. 5-24). Athens: Georgia.
4. Hammond, D. M. (1973). Life cycles and development of coccidia, p. 45-79. In D. M. Hammond and P. L. Long (ed.), The Coccidia: Eimeria, Isospora, Toxoplasma, and related genera. University Park Press, Baltimore, Md.

5. Innes, E.A. \& Vermeulen, A.N. (2006). Vaccination as a control strategy against the coccidial parasites Eimeria, Toxoplasma and Neospora. Parasitology, 133, S145-S168.

6. McDonald, V. \& Shirley, W.M. (2009). Past and future: vaccination against Eimeria. Parasitology, 136, 14771489.

7. Williams, R.B. (1999). A compartmentalized model for the estimation of the cost of coccidiosis to the world's chicken production industry. International Journal for Parasitology, 29, 1209-1229.

8. Reid, W.M. (1990). History of avian medicine in the United States. x. control of coccidiosis. Avian Diseases, 34, 509-525.

9. Williams, R.B. (2002). Anticoccidial vaccines for broiler chickens: pathways to success. Avian Pathology, 31, 317-353.

10. Vermeulen, A.N., Schaap, D.C. \& Schetters, Th.P.M. (2001). Control of coccidiosis in chickens by vaccination. Veterinary Parasitology, 100, 13-20.

11. Ruff, M.D. (1999). Important parasites in poultry production systems. Veterinary Parasitology, 84, 337347.

12. Vetterling, J.M. \& Doran, D.J. (1966). Schizogony and gametogony in the life cycle of the poultry coccidium, Eimeria acervulina Tyzzer, 1929. Journal of Parasitology, 52, 1150-1157.

13. Bhanushi, J.K. \& Long, P.L. (1985). Eimeria tenella infection: Does it affect humoral immune responses to heterologous antigens?. Journal of Parasitology, 71, 850-852.

14. McDougald, L.R. (2003). Coccidiosis. In Saif, Y.M., Barnes, H.J., Glisson, J.R., Fadley, A.M., McDougald, L.R. \& Swayne D.E (Eds.), Diseases of Poultry $\left(11^{\text {th }}\right.$ Edition) (pp. 974-1159). Imes: Iowa.

15. Lee, J.T., Broussard, D., Fitz-Coy, S., Burke, P., Eckert, N.H., Stevens, S.M., Anderson, P.N., Anderson, S.M. \& Caldwell, D.J. (2009). Evaluation of live oocyst vaccination or salinomycin for control of field-strain Eimeria challenge in broilers on two different feeding programs. Journal of Applied Poultry Research, 18, 458-464.

16. Dalloul, R.A. \& Lillehoj, H.S. (2005). Recent advances in immunomodulation and vaccination strategies against coccidiosis. Avian Diseases, 49, 1-8.

17. De Gussem, M. (2007). Pages 253-261 in proceedings of the $16^{\text {th }}$ European Symposium on Poultry Nutrition. (World Poultry Science Association). Strasbourg, France.

18. Martin, A.G., Danforth, H.D., Barta, J.R \& Fernando, M.A. (1997). Analysis of immunological cross- 
protection and sensitivities to anticoccidial drugs among five geographical and temporal strains of Eimeria maxima. International Journal for Parasitology, 27, 527-533.

19. Beattie, S.E. (1998). Immunity to and transport of sporozoites of Eimeria species in the domestic fowl (Gallus domesticus). University of Guelph MSc thesis, 3-12.

20. Lightowlers, M.W. (1994). Vaccination against animal parasites. Veterinary Parasitology, 54, 177-204.
21. Smith, A.L., Hesketh, P., Archer, A. \& Shirlery, M.W. (2002). Antigenic diversity in Eimeria maxima and the influence of host genetics and immunization schedule on cross-protective immunity. Infection and Immunity, 70, 2472-2479.

22. Chapman, H.D., Roberts, B., Shirley, M.W. \& Williams, R.B. (2005). Guidelines for evaluating the efficacy and safety of live anticoccidial vaccines, and obtaining approval for their use in chickens and turkeys. Avian Pathology, 34, 279-290. 